\title{
Treatment of Chemical Diabetes Mellitus With Sulfonylurea Compounds
}

\author{
By Stefan S. Fajans
}

\begin{abstract}
Abnormality in glucose tolerance appears to correlate with arteriosclerotic disease, even when the abnormality is quite mild. Though there is no evidence for prevention of these vascular problems by correction of the carbohydrate abnormality, it seems reasonable to attempt such a correction in
\end{abstract}

an investigational setting. We have used sulfonylurea (tolbutamide, chlorpropamide) in children and young adults (9-35 yr) without obesity, who have chemical diabetes. No apparent effect on insulin output has been noted, even with normalization of glucose tolerance.

R EFORE DISCUSSING the use of sulfonylurea compounds in the treatment of chemical diabetes, we must ask ourselves whether there is any evidence to indicate that mild carbohydrate intolerance may be harmful. Although this is a controversial question, there is some evidence that this may be true. There are four studies which give epidemiologic evidence. One is the Tecumsah, Michigan study. Dr. Ostrander, Dr. Epstein, and colleagues have shown that there is an excellent correlation between elevated blood sugar levels and the prevalence of coronary artery disease and generally of arteriosclerotic vascular disease. $^{1,2}$ This does not establish a cause and effect relationship necessarily, but it does establish a very strong correlation. Another epidemiologic study is the Bedford study, published by Keen et al. ${ }^{3,4}$ Individuals with chemical diabetes as well as those with what was termed "borderline diabetes" were compared with control subjects. "Borderline diabetics," by this definition, are individuals who had venous whole blood sugar levels between 120 and $200 \mathrm{mg} / 100 \mathrm{ml} 2 \mathrm{hr}$ after a $50-\mathrm{g}$ carbohydrate load. In these groups there was a correlation between blood sugar levels and the incidence of arterial disease. In addition to epidemiologic studies, there are studies of patients with arteriosclerotic heart disease. One study involved patients (mean age $46 \mathrm{yr}$ ) with coronary artery disease established by angiography. ${ }^{5}$ Abnormality of carbohydrate tolerance was found in $64 \%$ of those patients; abnormal lipid levels existed in about $51 \%$ of those patients. ${ }^{6}$ Combining both sets of observations, about $85 \%$ of all the patients with coronary heart disease had one or the other of these metabolic abnormalities. In another study of patients with coronary artery disease established by coronary arteriography, there was a $63 \%$ incidence of an abnormality of glucose tolerance, while $74 \%$ of the patients had an elevation of serum lipids. ${ }^{7}$ Abnormalities of carbohydrate or lipid metabolism were found in $91 \%$ of the patients.

From the Department of Internal Medicine, (Division of Endocrinology and Metabolism and the Metabolism Research Unit), University of Michigan, Ann Arbor, Mich.

Presented at the Conference on Chemical Diabetes Mellitus in Childhood, Ponte Vedra Beach, Florida, December 5-6, 1970.

Received for publication March, 1972.

Stefan S. Fajans, M.D.: Professor of Medicine, University of Michigan, Ann Arbor, Mich. 
Our own studies are anecdotal, but we have been impressed that, in families with arteriosclerotic vascular disease and diabetes, one observes the early appearance of coronary artery disease. We have the impression that many of the patients who develop coronary artery disease in middle age may have had some carbohydrate intolerance for many years. There is also some experimental evidence of a correlation between hyperglycemia and polyol formation in the aorta. ${ }^{8}$ Putting all these things together, it is my feeling that carbohydrate intolerance and hypoinsulinemia probably do lead to vascular damage. The internist sees patients with diabetes, be it mild or more severe, with cardiovascular disease, retinopathy, nephropathy, and with neuropathy. The pediatrician usually does not see these complications of diabetes. He is not as impressed with how mild the glucose intolerance can be in patients with these complications. Thus, it is easy to understand that the internist has been more concerned with prophylaxis than the pediatrician.

Nobody has data indicating that correction of carbohydrate intolerance in young individuals will prevent the occurrence of these complications; nevertheless, since these correlations exist in the adult it is reasonable to make an attempt to study this in the young. After all, we are not talking about established therapy, but we are discussing approaches to investigation. The investigative approaches do not imply that we recommend these procedures to every practicing physician in the country. We should make a very clear differentiation between investigative work and recommendations of what should be routine management.

Our interest in using sulfonylureas in chemical diabetes stems from a concept which prevailed in the 1950s, which I now believe to be incorrect. At that time it was thought that the course of diabetes in the young is normally one of rapid progression. In the early 1950s, in young adult individuals with carbohydrate intolerance that did not improve on diet alone, we used small doses of insulin in the hope of preventing the progression of diabetes. The sulfonylureas became available for investigation in 1955. I initiated sulfonylurea therapy in the first patient with chemical diabetes mellitus in 1957 when tolbutamide had become available generally for treatment of diabetes mellitus. This was a patient, aged 35 , who on repeated tests had had progression of carbohydrate intolerance. His tests reverted to normal after $4 \mathrm{mo}$ of therapy with tolbutamide. At that time the reason for using a sulfonylurea was not simply the greater convenience than using 10 or $15 \mathrm{U}$ of insulin. It was suspected, although not documented until the immunoassay for insulin became available, that one of the mechanisms of action of sulfonylureas was the increased secretion of insulin. As a result of the work of Loubatières with dogs, it was also thought that the use of the sulfonylureas might cause hypertrophy of the beta cells. Thus, we thought that we might be promoting an increase in beta-cell mass in these individuals. According to information we have published in the past, in a considerable number of individuais who were between the ages of 9 and $35 \mathrm{yr}$ at diagnosis, we did have a certain amount of success in improving carbohydrate tolerance with the use of sulfonylureas. ${ }^{9-11}$ Later, with the availability of immunoassays for insulin, we did not 
find improvement in the insulin response to glucose. However, there may be an increased effectiveness of circulating insulin, since blood glucose concentrations are lower. Although I have great reservations about applying insulin/ glucose ratios to individual cases, it may be useful in evaluating the response to therapy of groups of individuals.

I still think that sulfonylureas have something to offer in improving carbohydrate tolerance in chemical diabetes. However, I am not as sure today as I was $5 \mathrm{yr}$ ago, because we have learned more about the natural history of the disease. When we administered tolbutamide to the first patient with chemical diabetes $13 \mathrm{yr}$ ago, my concept was that we were dealing with a rapidly progressive disease. It is now clear that this is frequently not the case when we establish the diagnosis of asymptomatic diabetes. ${ }^{12}$

As I discussed earlier, on successive glucose tolerance tests, whether looking at levels of blood glucose or plasma insulin, there may be marked fluctuations. It is unwise to draw any conclusions on the basis of a few glucose tolerance tests done before and after sulfonylurea therapy. Sequential testing is essential in order to develop reliable observations.

Despite variability in the course of chemical diabetes in the young, I am still under the impression that in some patients the sulfonylureas have a favorable effect on carbohydrate tolerance. In our studies, some of the patients that we switched to sulfonylureas had been on diet or diet plus insulin for $5 \mathrm{yr}$. Each of these patients served as his own control. In the last $10 \mathrm{yr}, \mathrm{I}$ have started a patient on a sulfonylurea only when he had at least four glucose tolerance tests at 3-mo intervals in which either no improvement on diet or progressive intolerance on diet alone had been shown. Thus, in each patient, we have established that there is not spontaneous fluctuation or improvement in carbohydrate tolerance prior to initiation of sulfonylurea therapy.

We perform glucose tolerance tests with the patient taking the sulfonylurea until the night before, and then we do the second glucose tolerance test after 2 days off tolbutamide or $2 \mathrm{wk}$ off chlorpropamide. The classification of the status of each group is not on the basis of a single test but on the basis of multiple tests. All of these patients, of course, are nonobese. We have not administered these agents to any obese children or young adults; they are managed with diet alone.

In summary, our results with sulfonylurea therapy are as reported previously: There is a group that has shown no improvement, a few of whom have gone on to insulin requirement; there is a group that has shown a definite improvement in carbohydrate tolerance; and there is a small group that actually has had complete normalization of carbohydrate tolerance.

\section{REFERENCES}

1. Ostrander, L. D., Jr., Francis, T., Jr., Hayner, N. S., Kjelsberg, M. O., and Epstein, F. H.: The relationship of cardiovascular disease to hyperglycemia. Ann. Intern. Med. 62:1188, 1965.

2. Epstein, F. H.: Hyperglycemia. A risk factor in coronary heart disease. Circulation $36: 609,1967$.

3. Keen, H., Rose, G., Pyke, D. A., Boyns, D., Chlouverakis, C., and Mistry, S.: Blood sugar and arterial disease. Lancet 2:505, 1965. 
4. - , and Jarrett, R. J.: The effect of carbohydrate tolerance on plasma lipids and atherosclerosis in man. In Jones, R. J. (Ed.): Artherosclerosis: Proceedings of the Second International Symposium. Berlin, Springer-Verlag, 1970, p. 435.

5. Herman, M. V., and Gorlin, R.: CoroIn Hamwi, G. J., and Danowski, T. S. (Eds.): Diabetes Mellitus: Diagnosis and Treatment, Vol. II. New York, Academic, 1967, p. 185.

6. Heinle, R. A., Fredrickson, D., Levy, R. I., Herman, M. V., and Gorlin, R.: Incidence of metabolic abnormalities in angiographically demonstrated coronary heart disease. J. Clin. Invest. 46:1069, 1967.

7. Falsetti, H. L., Schnatz, J. D., Greene, D. G., and Bunnell, I. L.: Serum lipids and glucose tolerance in angiographically proved coronary artery disease. Chest 58:111, 1970.

8. Morrison, A. D., and Winegard, A. I. Regulation of polyol pathway activity in aorta. Diabetes 20:329, 1971.

9. Fajans, S. S., and Conn, J. W.: Tolbutamide-induced improvement in carbohydrate tolerance of young people with mild diabetes mellitus. Diabetes 9:83, 1960 .

10. - , and - : The use of tolbutamide in the treatment of young people with mild diabetes mellitus-A progress report. Diabetes $11: 123,1962$.

11. - , and --: Prediabetes, subclinical diabetes and latent clinical diabetes: Interpretation, diagnosis and treatment. In Leibel B. 5., and Wrenshall, B. A. (Ed5.): On the Nature and Treatment of Diabetes. International Congress Series 84. New York Excerpta Medica Foundation, 1965, p. 641. 12. --, Floyd, J. C., Jr., Pek, S., and Conn, J. W.: The course of asymptomatic diabetes in young people, as determined by levels of blood glucose and plasma insulin. Trans. Assoc. Am. Physicians 82:211, 1969. 